international collaboration and building capacity in guideline development methodology and implementation.

\section{P196 UPDATING THE EVIDENCE FOR CPGS: THE USEFULNESS OF RSS FEEDS}

${ }^{1} \mathrm{I}$ Etxeandia-lkobaltzeta, ${ }^{1} \mathrm{~N}$ Ibargoyen-Roteta, ${ }^{2} \mathrm{R}$ Roteche-del Campo, ${ }^{3} \mathrm{~A}$ Etxeberria-Agirre, ${ }^{4} \mathrm{M}$ Callén-Blecua. 'Basque Office for Health Technology Assessment (OSTEBA)," VitoriaGasteiz, Spain; ${ }^{2}$ Primary Care Centre of Alza (Osakidetza - NHS), Alza, Spain; ${ }^{3}$ Primary Care Centre of Hernani (Osakidetza - NHS), Hernani, Spain; ${ }^{4}$ Primary Care Centre of Bidebieta (Osakidetza - NHS), Bidebieta, Spain

\section{0:1136/bmjqs-2013-002293.201}

Background Scientific knowledge is in constant evolution and even if Clinical Practice Guidelines (CPGs) get out of date in five years time, there is a lack of standardised method for an efficient updating process.

Context A proposal used to update efficiently the bibliographic searches during the development of a CPG on childhood asthma management is described.

Description of Best Practice Bibliographic searches were performed in Medline, Embase and All EBM databases using Ovid, and in BMJ update database. Search strategies and RSS-feeds were created and saved when possible. E-mail alerts for those searches carried out in databases with no RSS services were also created. All the RSS-feeds were centralised in a Netvibes web portal and organised into tabs (one tab by clinical question). New alerts were revised periodically during the guideline development process and new references incorporated in the guideline when relevant. RSS-feeds could be created for Medline and Embase searches. The centralization of RSS-feeds in a Netvibes portal facilitated the reviewing process of the latest published articles by clinical question. The review of the email-alert services generated for those sources with no RSS services was more time-consuming. Lessons-for-Guideline-Developers-Adaptors-Implementers-and/orUsers The management of RSS-feeds is considered as a more effective tool to check and follow all the new references published during the guideline development process, being useful also after the CPG publication. Nevertheless, not all databases allowed the creation of RSS feeds, an issue that should be considered by their managers, and in all cases, the updating process has to be supported by professionals.

\section{P198 ADHERENCE TO INFERTILITY GUIDELINES WITH REGARD TO TREATMENT POLICY}

${ }^{1} \mathrm{~F}$ Kersten, ${ }^{1} \mathrm{~W}$ Nelen, ${ }^{2} \mathrm{M}$ Goddijn, ${ }^{1} \mathrm{D}$ Braat, ${ }^{2} \mathrm{~B}$ Mol, R Hermens. ${ }^{1}$ Department of Obstetrics and Gynaecology, Radboud University Nijmegen Medical Centre, Nijmegen, The Netherlands; 'Centre for Reproductive Medicine, Academic Medical Centre, Amsterdam, The Netherlands; ${ }^{3}$ IQ Healthcare, Radboud University Nijmegen Medical Centre, Nijmegen, The Netherlands

\section{0:1136/bmjqs-2013-002293.202}

Background Nowadays, prognostic models are increasingly being used in reproductive medicine. One of these models calculates the chances of conception for couples with unexplained infertility and subsequently indicates the right treatment policy. The Dutch national network guideline on infertility recommends the use of this prognostic model to prevent unnecessary treatment. This strategy is supported by international guidelines on the management of infertility (ESHRE and NICE).

Objectives The aim of this study is to evaluate guideline adherence regarding treatment policy for couples with unexplained infertility in 25 Dutch clinics.
Methods In this retrospective study, we assessed guideline adherence with regard to 1) the use of the prognostic model and 2) the right treatment decision. Data were collected from medical records.

Results In this abstract we present the preliminary data of ten clinics. At the GIN conference we will have the results of all 25 participating clinics. So far we included 208 couples with unexplained infertility. In $54 \%$ of the couples the prognostic model was used. In $69 \%$ of the couples the right treatment decision was primarily made. However, ultimately, $41 \%$ of all couples received unnecessary treatment.

Discussion This study shows that adherence to the guideline regarding treatment policy is not optimal. Despite the guideline recommendations, more than one third of couples are exposed to unnecessary treatment and risks.

Implications for Guideline Developers/Users Implementation of the guideline is not optimal. To improve guideline implementation a multifaceted strategy has to be developed and evaluated.

\section{P199 THE CHALLENGES FOR AN ALCOHOL AND DRUG EDUCATION GUIDELINE FOR COMMUNITY HEALTH WORKERS}

$1,2,3 \mathrm{~A}$ Stein, ${ }^{4} \mathrm{~A}$ Fernandes, ${ }^{5} \mathrm{C}$ Giugliani. ${ }^{1}$ Conceicao Hospital, Porto Alegre, Brazil; ${ }^{2}$ Ufcspa, Porto Alegre, Brazil; ${ }^{3}$ Ulbra, Canoas, Brazil; ${ }^{4}$ Ufrgs, Porto Alegre, Brazil; ${ }^{5}$ Ufrgs, Porto Alegre, Brazil

\section{0:1136/bmjqs-2013-002293.203}

Introduction There is a need to train and develop a guideline for Community Health Workers (CHW) on how to tackle alcohol and drugs problem.

Objective To identify the profile and mental health problems of $\mathrm{CHW}$ and how they manage drug problem.

Methodology A descriptive study was performed and data collected from all $\mathrm{CHW}$ who work at Primary Health Care of Passo Fundo (Brazil). A Self Report Questionnaire (SRQ) was collected to screen mental health on $\mathrm{CHW}$ and scores higher than 7 were identified as positive for mental health problem. Their knowledge about drugs was also collected using a standard questionnaire. The Project was approved by the Passo Fundo University IRB. Descriptive statistics were applied.

Results All $78 \mathrm{CHW}$ were women, age $41.1 \pm 9.6$ years old, $57.7 \%$ have completed high school level, $69.2 \%$ were married and $98.7 \%$ were from lower social class. Half of the CHW were sedentaries, 32\% were smokers, 93.6\% did not report alcohol dependency, although 59\% reported to have a member of their family as having drug or an alcohol problem. They reported that alcohol and drug is a major problem in the community. There were $29.5 \%$ of CHW who have SRQ score higher than 7 . The mean grade of the knowledge test was 34\%.

Conclusion There is a need to develop a guideline on alcohol and drugs for CHW. The profile of CHW had shown a high level of psychiatric problems and lack of basic information.

\section{P200 SUICIDE RISK ASSESSMENT ACCORDING TO BEST PRACTICE GUIDELINES: THE DEVELOPMENT OF A CHART AUDIT PERFORMANCE MEASURE}

${ }^{1}$ E Santa Mina, ${ }^{2} \mathrm{C}$ O'Grady, ${ }^{1} \mathrm{E}$ McCay. ${ }^{1}$ Ryerson University, Toronto, Canada; ${ }^{2}$ Centre for Addiction and Mental Health, Toronto, Canada; ${ }^{3}$ Ryerson University, Toronto, Canada

10:1136/bmjqs-2013-002293.204 
Background Best practice guidelines (BPGs) in suicide risk assessment documentation support nursing care of clients at risk for suicide. Investigation regarding nurses' adherence to BPGs for suicide risk assessment documentation is minimal.

Objectives In a mixed-methods study to investigate nurses' knowledge of suicide risk assessment documentation, the researchers created a chart audit to measure nursing practice congruence with five recommendations from the suicide risk assessment BPG (RNAO, 2009).

Methods Five recommendations, from the BPG: Assessment and Care of Adults at Risk for Suicidal Ideation and Behaviour (RNAO, 2009), were the benchmarks for the chart audit measure. Suicide risk indicators, as determined by the Minimum Data Set for Mental Health (MDS-MH) (Ontario Ministry of Health, 2011), were the criteria to identify charts of suicidal clients. The researchers integrated MDS-MH indicators with the five BPG recommendations and constructed compliance indicators that incorporated the Nurses Global Assessment of Suicide Risk (Cutcliffe \& Barker, 2004).

Results Five BPG recommendations, integrated with provincial suicide assessment criteria and a standardised suicide assessment scale produced a 3-point likert scale chart audit with 15 indicators. Possible ranges of scores for documentation congruence with the BPG are 0 to 30 .

Discussion This performance measure provides objective, proxy data to triangulate with nurses' self-perception of suicide risk documentation and evaluate practice as per BPGs.

Implications for Guideline Developers/Users A standardised instrument to monitor BPG practices can be used to inform implementation and education strategies.

\section{P204 THE UPDATING PROCESS GUIDANCE IN CLINICAL PRACTICE GUIDELINES HANDBOOKS: A SYSTEMATIC REVIEW}

1,2,3R Vernooij, 2,3A Sanabria, 2,3 Solà, 2,3 P Alonso-Coello, 2,3 L Martínez García. ${ }^{1}$ Department of Health Sciences, Faculty of Earth and Life Sciences, VU University, Amsterdam; ${ }^{2}$ The Netherlands, Iberoamerican Cochrane Centre, Barcelona, Spain; ${ }^{3}$ Institute of Biomedical Research (IIB Sant Pau), Barcelona, Spain

\section{0:1136/bmjqs-2013-002293.205}

Background Updating is an important process for maintaining recommendations' and clinical practice guidelines' (CPGs) validity. CPGs methodological handbooks are designed to provide guidance on developing and updating CPGs, however, little is known about this guidance about the updating process.

Objectives To identify and describe the guidance about the updating process in CPGs handbooks.

Methods We included methodological handbooks that provide guidance about updating. We conducted a systematic search in the Guidelines International Network library, US National Guidelines Clearinghouse, MEDLINE and contacted main institutions. For extracting data we developed a list of key elements. Results We included thirty-six handbooks. Most of them (97\%) focus mainly on developing de novo CPGs and include some information about updating. Half of the handbooks provide a time frame for updating guidelines. The majority of handbooks do not provide guidance about the updating process, namely: literature search, evidence selection, assessment, synthesis and external review. Finally, two handbooks (6\%) provide information about publishing an updated CPG.

Discussion Our study highlights that the updating process is poorly described in current methodological handbooks.
Handbooks do not contain enough information for executing an optimal update.

Implications Institutions responsible for updating and developing CPGs need to pay more attention to updating and reflect this in their handbooks. This guidance should be more rigorous, explicit and detailed. This could, consequently, lead to a more optimal updating process and, hence, more up-to-date recommendations.

\section{P207 TOOLKIT: IMPLEMENTATION OF BEST PRACTICE GUIDELINES - A FRAMEWORK FOR SUCCESS!}

H McConnell, A Stewart-Pyne, I Bajnok. Registered Nurses' Association of Ontario, Toronto, Canada

\section{0:1136/bmjqs-2013-002293.206}

A signature programme lead by a professional nursing association in Canada has a mandate to develop, disseminate, implement, evaluate and support the uptake of clinical guidelines. This programme's success has resulted in international reach, due to its rigorous guideline development process, and innovative implementation strategies. These strategies include a key resource, the Toolkit: Implementation of Best Practice Guidelines, which delineates a systematic, well-planned implementation process, and is designed to assist nurses and other healthcare professionals to support evidence-informed clinical decisionmaking. This Toolkit is based on emerging evidence that the likelihood of achieving successful uptake of best practice in health care increases when: - Guidelines are selected for implementation through systematic, participatory processes including relevant stakeholder engagement and environmental readiness assessment - Guidelines are tailored to the local context - Barriers and facilitators to guideline use are assessed and addressed - Guideline use is systematically monitored and sustained Evaluation of the impacts of guideline use is an integral part of the entire process - There are adequate resources to support completion of all aspects of implementation This Toolkit will help guideline users take best evidence and integrate it into practice, education and policy using a systematic approach consistent with the local context of practice. This presentation will share the key phases of guideline implementation outlined in the Toolkit, and discuss how this resource is being utilised to address the key challenges of developing evidence based practice cultures through guideline implementation.

\section{P208 HOW COULD WHO BETTER SUPPORT NATIONAL AND SUBNATIONAL GOVERNMENTS IN THEIR EFFORTS TO ADAPT AND IMPLEMENT GLOBAL RECOMMENDATIONS AND DECISIONS? A SYSTEMATIC ANALYSIS OF HEALTH SYSTEMS GUIDANCE AND WORLD HEALTH ASSEMBLY RESOLUTIONS}

${ }^{1,2} \mathrm{D}$ Patino-Lugo, '1 Lavis, ${ }^{3} \mathrm{P}$ Perel, ${ }^{1} \mathrm{Y}$ Wu, ${ }^{3} \mathrm{~A}$ Haines, ${ }^{4} \mathrm{M}$ Ranson, ${ }^{5} \mathrm{U}$ Panisset, ${ }^{6} \mathrm{X}$ Bosch-Capblanch, ${ }^{1} \mathrm{M}$ Brouwers. ${ }^{1} \mathrm{McM}$ aster University, Hamilton, Canada; ${ }^{2}$ University of Antioquia, Medellin, Colombia; ${ }^{3}$ London School of Hygiene and Tropical Medicine, London, UK; ${ }^{4}$ Alliance for Health Policy and Systems Research, WHO, Geneva, Switzerland; ${ }^{5}$ Evidence and Networks for Health - EVIPNet, WHO, Geneva, Switzerland; ${ }^{6}$ Swiss Tropical and Public Health Institute Basel, Switzerland

\section{0:1136/bmjas-2013-002293.207}

Background The World Health Organization's health systems guidance and the normative standards about health systems 